\title{
A NONLOCAL OPERATOR BREAKING THE KELLER-OSSERMAN CONDITION
}

\author{
RAÚL FERREIRA AND MAYTE PÉREZ-LLANOS
}

\begin{abstract}
This work is concerned about the existence of solutions to the nonlocal semilinear problem

$$
\begin{cases}-\int_{\mathbb{R}^{N}} J(x-y)(u(y)-u(x)) d y+h(u(x))=f(x) & x \in \Omega, \\ u=g & x \in \mathbb{R}^{N} \backslash \Omega,\end{cases}
$$

verifying that $\lim _{x \rightarrow \partial \Omega, x \in \Omega} u(x)=+\infty$, known in the literature as large solutions. We find out that the relation between the diffusion and the absorption term is not enough to ensure such existence, not even assuming that the boundary datum $g$ blows up close to $\partial \Omega$. On the contrary, the role to obtain large solutions is played only by the interior source $f$, which gives rise to large solutions even without the presence of the absorption. We determine necessary and sufficient conditions on $f$ providing large solutions, compute the blow-up rates of such solutions in terms of $h$ and $f$. Finally, we also study uniqueness of large solutions.
\end{abstract}

\section{INTRODUCTION}

In this work we analyze the existence of large solutions to the following semilinear nonlocal problem

$$
\begin{cases}-\int_{\mathbb{R}^{N}} J(x-y)(u(y)-u(x)) d y+h(u(x))=f(x) & x \in \Omega, \\ u=g & x \in \mathbb{R}^{N} \backslash \Omega .\end{cases}
$$

Here the kernel $J$ is a smooth probability density, $f$ is a continuous function, $g \in L^{1}\left(\mathbb{R}^{N} \backslash \Omega\right)$ and $h$ is an increasing continuous function.

By large solutions we understand solutions satisfying

$$
\lim _{x \rightarrow \partial \Omega, x \in \Omega} u(x)=+\infty .
$$

This interpretation of large solutions was originated by the works of Keller and Osserman, see [6] and [9], who separately proved that the Cauchy problem $-\Delta u+h(u)=0$ has not entire solutions (solutions well defined in the whole $\left.\mathbb{R}^{N}\right)$ if $h$ is non decreasing and verifies

$$
\int_{0}^{\infty}\left(\int_{0}^{x} h(z) d z\right)^{\frac{-1}{2}} d x<\infty
$$

2010 Mathematics Subject Classification: 45P05, 35B40, 35J61.

Keywords: Nonlocal diffusion, Large solutions, Keller-Osserman condition. 
Furthermore, Keller shows that in this case, for any bounded domain, $\Omega$, there exists a solution to $-\Delta u+h(u)=0$ in $\Omega$ such that (2) holds. Condition (3) is known as Keller-Osserman condition and in fact it is a necessary and sufficient condition if in addition $h \in C^{1}([0,+\infty))$ and $h(0)=0$.

Existence of this kind of solutions has been analyzed by a large number of authors for a wide variety of diffusions and nonlinearities, see for instance the compiling paper by C. Bandle and M. Marcus [1], the books by J. LópezGómez [7] and L. Véron [11] and references therein.

In [3] the authors use the monotonicity methods developed in [8] to study large solutions to the problem

$$
\begin{cases}\Delta u=\lambda \frac{f(u)}{1+\frac{1}{|\Omega|} \int_{\Omega} g(u) d x} & x \in \Omega, \\ u=\infty & x \in \partial \Omega .\end{cases}
$$

Notice the nonlocal character of the above absorption term. However, the problem under consideration here corresponds to a nonlocal diffusion operator, competing with a local absorption given by the function $h(u)$.

On the other hand, nonlocal diffusion problems have attracted a great interest in the last years, though within this context of large solutions, the literature is not so rich, to our knowledge. In [2] the authors change the diffusion by the fractional laplacian and consider the absorption $h(u)=$ $|u|^{p-1} u$. Precisely, they study existence of large solutions to

$$
\begin{cases}-\int_{\mathbb{R}^{N}} \frac{u(x+y)-u(x)}{|y|^{2 \alpha+N}} d y+|u|^{p-1} u=0 & x \in \Omega, \\ u=0 & x \in \mathbb{R}^{N} \backslash \Omega,\end{cases}
$$

with $\alpha \in(0,1)$. They prove that if $p \in\left(1+2 \alpha, p_{*}(\alpha)\right)$ there exists a unique large solution, whose precise asymptotic behaviour close to the boundary is given by $\operatorname{dist}(x, \partial \Omega)^{-\gamma}$, with $\gamma=2 \alpha /(p-1)$.

In [10] it is shown existence and uniqueness of large solutions for the problem

$$
\int_{|y| \leq \rho(x)} \frac{u(x+y)-u(x)}{|y|^{2 \alpha+N}} d y+|u|^{p-1} u=0 \quad x \in \Omega
$$

where $\rho(x)=\Lambda \operatorname{dist}(x, \partial \Omega)^{\sigma}$ with $0<\Lambda<1$. Since $\Lambda<1$ the integration is performed within $\Omega$, thus no condition is needed in the complementary set. They prove that for certain relations of the parameters $\alpha, \sigma, \Lambda$ and $p$ there exists a unique large solution, which approaching the boundary behaves as $\operatorname{dist}(x, \partial \Omega)^{-\frac{\sigma(\alpha-2)+2}{p-1}}$.

We wish to emphasize here that in both of the mentioned problems, the authors consider a singular symmetric kernel, while our kernel is smooth and not necessarily symmetric. Furthermore, the operator associated to our kernel (which is integrable) is not even of differential nature, contrary to what happens for the fractional Laplacian. On the contrary, if $J$ is integrable we have a zero-order operator competing with a nonlinear term 
$h(u)$. In our opinion, that is precisely the reason behind the breakage of the Keller-Osserman condition. An interesting question arising here is if being integrable is a necessary and sufficient requirement on $J$ for the rupture of the Keller-Osserman condition. In the last Section we answer part of the question for symmetric kernels belonging to $L^{1}\left(\mathbb{R}^{n}\right)$.

If we rewrite Problem (1) in the form

$$
-\int_{\Omega} J(x-y) u(y) d y+u(x)+h(u(x))=f(x)+\int_{\mathbb{R}^{N} \backslash \Omega} J(x-y) g(y) d y,
$$

it is clear that the datum $g$ does not play any role for existence of large solutions. Recall that $g \in L^{1}\left(\mathbb{R}^{N} \backslash \Omega\right)$, hence the last term is bounded thus not relevant in the analysis of the blow-up. On the other hand, this hypothesis on $g$ is essential to give sense to the equation.

Now, we seek large solutions for the corresponding Keller-Osserman problem

$$
-\int_{\Omega} J(x-y) u(y) d y+u(x)+h(u(x))=0 .
$$

We will see that any continuous large solution must be bounded from below and $u \notin L^{1}(\Omega)$. As a result the convolution term does not make sense and none large solution exists. This is the core of the following Theorem:

Theorem 1.1. Let $f$ be a continuous bounded function, $g \in L^{1}\left(\mathbb{R}^{N} \backslash \Omega\right)$ and $h$ continuous and increasing. Then, there does not exist any continuous large solution for Problem (1).

According to this Theorem, it does not exist any absorption term, capable of compensating this kind of nonlocal diffusion, if we wish that (2) holds. This fact, not only exhibits another important difference concerning the behaviour of solutions to this kind of nonlocal problems with respect to models involving non localities of fractional type. It constitutes indeed, a notorious novel result within the theory of large solutions.

In consequence, if we look for large solutions to our problem, the function $f$ must be required from now on to satisfy

$$
\lim _{x \rightarrow \partial \Omega, x \in \Omega} f(x)=+\infty .
$$

Let us determine now a necessary and sufficient condition replacing (3) for our problem. For this purpose, it will be useful to introduce the function

$$
H(s)= \begin{cases}h^{-1}(s) & \text { if } \frac{h(s)}{s} \rightarrow \infty \text { as } s \rightarrow \infty, \\ s & \text { if } \frac{h(s)}{s} \text { is bounded, }\end{cases}
$$

to unify the subsequent notation. 
Turning back our attention to equation (5), it is clear that the terms $u$ and $h(u)$ compete for the role of providing large solutions. More concisely, we show

Theorem 1.2. Let $g \in L^{1}\left(\mathbb{R}^{N} \backslash \Omega\right)$, $f$ be a continuous function verifying (6), $h$ a continuous and increasing function and $H$ defined in (7). Then Problem (1) admits large solutions if and only if

$$
\int_{\Omega} H(f(x)) d x \leq C .
$$

Remark 1.1. We note that, contrarily to what occurs for local diffusions, the presence of the term $u$ in the equation allows to obtain large solutions, even when the absorption is null or bounded. We would like to emphasize the surprising difference with respect to its local linear counterpart $-\Delta u(x)=f(x)$ if $x \in \Omega$ and $u=\infty$ on $\partial \Omega$, for which no solution exists under assumption (6).

Our second result deals with the blow-up rate for large solutions obtained as an approximation procedure. This approach returns the minimal large solution $u$ in the sense that any large solution $v$ satisfies $v \geq u$. Regarding the maximal solution, for local problems it is usually constructed as the limit of large solutions to the problem settled in certain subdomain $\Omega_{\varepsilon} \subset \subset \Omega$. However, in our case $f$ is bounded in $\Omega_{\varepsilon}$ and by Theorem 1.1 no large solution exists in $\Omega_{\varepsilon}$. For this reason, we need to consider a family of functions $f_{\varepsilon}$ that blow up on the boundary $\partial \Omega_{\varepsilon}$, to guarantee the existence of large solutions for the approximating problem, see Remark 3.3 below.

Theorem 1.3. Let $u$ be a large solution. Then, there exist two positive constants such that

$$
H(\delta f(x))-C_{1} \leq u(x) \leq H(f(x))+C_{2},
$$

where the parameter $\delta$ is given by

$$
\delta= \begin{cases}1 & \text { if } \frac{h(s)}{s} \rightarrow \infty \quad \text { as } s \rightarrow \infty, \\ \frac{1}{1+C} & \text { if } \frac{h(s)}{s}<C .\end{cases}
$$

Now we focus on the uniqueness of large solutions. Accordingly to the hypothesis of $h$ and $J$, we perform two different arguments to accomplish the uniqueness result.

Theorem 1.4. In the hypothesis of Theorem 1.2. If either $f \geq 0$ and the function $s \rightarrow h(s) / s$ is unbounded and nondecreasing, or, $J$ is a symmetric function then the large solution is unique.

Remark 1.2. A typical example of explosive source is $f=\operatorname{dist}(x, \partial \Omega)^{-\gamma}$. In this case, if we take $h(s)=|s|^{p-1} s$ by condition (8) there exist large solutions if and only if $\gamma<r:=\max (1, p)$. Furthermore, close to the boundary

$$
u \sim \operatorname{dist}(x, \partial \Omega)^{-\frac{\gamma}{r}} .
$$


However, if we choose $h(s)=e^{s}$ there are no large solutions, since the condition (8) reads

$$
\int_{\Omega} H(f(x)) d x=-\gamma \int_{\Omega} \log (\operatorname{dist}(x, \partial \Omega)) d x=\infty .
$$

This last example illustrates another important difference with respect to the (local) laplacian, which admits large solutions with exponential absorption.

Remark 1.3. Following step by step the proofs of the above theorems, a similar result can be established if we assume that $f$ blows up only at certain $\Gamma \subset \partial \Omega$.

In this case there exist large solutions if and only if (8) holds. Moreover, $u$ blows up only at $\Gamma$, with blow-up rate given also by (9).

Let us conclude this introduction specifying which is the notion of solution that we are using along the paper:

Definition 1.1. We say that $u$ is a classical solution of $(1)$ if $u \in C(\Omega)$ and satisfies (1) pointwise.

This paper is organized as follows. In the next section we show that there does not exist any large solution to (1) whenever $f$ is bounded, namely we prove Theorem 1.1. Section 3 includes some preliminary results on existence and comparison of solutions when $f$ is bounded. Then we prove Theorem 1.2 with the use of approximation arguments. Section 4 contains our uniqueness results and finally, the last section is devoted to extend the breakage of the Keller- Osserman condition to integrable and symmetric kernels.

\section{Breakage of the Keller-Osserman condition}

We devote this section to prove Theorem 1.1. First, we make the following observations:

(1) Any classical large solution is bounded from below. Notice that it is a continuous function diverging to infinity as $x$ approaches the boundary.

(2) Moreover, if $f$ is a bounded function then, none classical large solution of (1) belongs to $L^{1}(\Omega)$. In fact, assuming that $u \in L^{1}(\Omega)$, from (5) we infer that

$$
\begin{aligned}
u(x)+h(u(x)) & =f(x)+\int_{\mathbb{R}^{N} \backslash \Omega} J(x-y) g(y) d y+\int_{\Omega} J(x-y) u(y) d y \\
& \leq\|f\|_{L^{\infty}(\Omega)}+\|J\|_{L^{\infty}\left(\mathbb{R}^{N}\right)}\|g\|_{L^{1}\left(\mathbb{R}^{N} \backslash \Omega\right)}+\|J\|_{L^{\infty}\left(\mathbb{R}^{N}\right)}\|u\|_{L^{1}(\Omega)} .
\end{aligned}
$$

Since the function $s \rightarrow s+h(s)$ is increasing and it goes to infinity, the above estimate turns impossible the occurrence of (2).

Ad contrarium let us admit that a classical large solution $u$ exists. From point two above we know that necessarily $u \notin L^{1}(\Omega)$. This fact means that 
there must exist a point $x_{0} \in \partial \Omega$ such that

$$
\int_{\Omega \cap B_{\delta}\left(x_{0}\right)} u(y) d y=\infty
$$

for some $\delta>0$. Let us fix $x \in \Omega \cap B_{\delta}\left(x_{0}\right)$ and $\delta$ small enough ensuring that

$$
J(x-y) \geq \alpha>0, \quad y \in \Omega \cap B_{\delta}\left(x_{0}\right) .
$$

Taking into account that $u$ is bounded from below, we obtain that

$$
\begin{aligned}
\int_{\Omega} J(x-y) u(y) d y & =\int_{\Omega \backslash\left(\Omega \cap B_{\delta}\left(x_{0}\right)\right)} J(x-y) u(y) d y+\int_{\Omega \cap B_{\delta}\left(x_{0}\right)} J(x-y) u(y) d y \\
& \geq-C\|J\|_{L^{1}\left(\mathbb{R}^{n}\right)}+\alpha \int_{\Omega \cap B_{\delta}\left(x_{0}\right)} u(y) d y=\infty .
\end{aligned}
$$

Recalling (5), this implies that $u(x)=\infty$, hence no large solution exists.

Remark 2.1. Notice that the argument above does not require the continuity of $J$. Then, if the kernel $J \in L^{1}\left(\mathbb{R}^{n}\right)$ satifies (10) any large solution must belong to $L^{1}(\Omega)$.

\section{Existence OF LARGE SOLUTIONS}

From now on, we will turn our attention to precise necessary and sufficient conditions for existence of large solutions. This aim will be accomplished by approximation arguments. Therefore, we start by showing existence of (bounded) solutions when $f$ is bounded, via the sub-supersolution method. Thus, we first prove a comparison result.

Lemma 3.1. Let $\bar{u}$ and $\underline{u}$ be a classical bounded supersolution and subsolution, respectively. Then, $\underline{u} \leq \bar{u}$.

Proof. Define $w(x)=\underline{u}(x)-\bar{u}(x)$, we get that

$-\int_{\Omega} J(x-y)(w(y)-w(x)) d y+w(x) \int_{\mathbb{R}^{N} \backslash \Omega} J(x-y) d y+h(\underline{u}(x))-h(\bar{u}(x)) \leq 0$.

In order to get a contradiction we define $K=\sup _{\Omega} w$ and assume that $K>0$.

i) If there exists $x_{0} \in \Omega$ such that $w\left(x_{0}\right)=K$, then evaluating the previous expression at $x_{0}$, we observe that the first two terms are non-negative. Hence $h\left(\underline{u}\left(x_{0}\right)\right)-h\left(\bar{u}\left(x_{0}\right)\right) \leq 0$. Applying the monotonicity of $h$ leads to the desired contradiction.

ii) Now we admite that there exists a sequence $x_{n} \rightarrow x \in \partial \Omega$, such that $w\left(x_{k}\right) \rightarrow K$. Using the dominate convergence theorem and the fact that $w$ is bounded we can pass to the limit in the previous expression to infer

$-\int_{\Omega} J\left(x_{0}-y\right)(w(y)-K) d y+K \int_{\mathbb{R}^{N} \backslash \Omega} J(x-y) d y+h\left(\underline{u}\left(x_{0}\right)\right)-h\left(\bar{u}\left(x_{0}\right)\right) \leq 0$.

The contradiction follows now arguing as in the previous step. 
Remark 3.1. We point out that the comparison Lemma also holds even assuming that $\bar{u} \rightarrow \infty$ as $x \rightarrow \partial \Omega$.

The solution to the following problem will be useful to construct suitable sub and supersolutions to (1), when $f$ is bounded.

Lemma 3.2. There exists a classical non-negative bounded solution of

$$
-\int_{\Omega} J(x-y) w(y) d y+w(x)=1 .
$$

Proof. The existence is obtained by the sub-supersolution method. First, we note that $\underline{w}=0$ is a subsolution. To find a positive supersolution we consider two cases.

i) $J$ has compact support. Define $\bar{w}=k \phi_{1}$, where $\phi_{1}$ is the first eigenfunction of the operator

$$
\mathcal{L}=-\int_{\Omega} J(x-y) u(y) d x+u(x)
$$

which is continuous and strictly positive in $\bar{\Omega}$, see [5]. Since $\phi(x)>0$ in $\bar{\Omega}$, we can take $k$ large enough to get

$$
-\int_{\Omega} J(x-y) \bar{w}(y) d y+\bar{w}(x)=\lambda_{1} k \bar{w}(x)>1 .
$$

ii) If the support of $J$ is unbounded we take $\bar{w}=k$. Notice that since $\Omega$ is bounded

$$
\sup _{x \in \Omega} \int_{\Omega} J(x-y) d y=\eta<1 .
$$

Then, if $k$ is large enough we have

$$
-\int_{\Omega} J(x-y) \bar{w}(y) d y+\bar{w}(x)=-\eta k+k \geq 1 .
$$

Theorem 3.1. Let $f$ be a continuous bounded function. Then there exists a bounded classical solution of (1).

Proof. Let $w$ be the function given in the previous Lemma. We claim that the functions

$$
\bar{u}(x)=\lambda w(x), \quad \underline{u}(x)=-\mu w(x)
$$

being $\lambda$ and $\mu$ positive parameters, are a supersolution and a subsolution to Problem (1), respectively.

Moreover, clearly $\underline{u} \leq \bar{u}$, and hence the sub-supersolution method (see [4]) guarantees the existence of a solution such that

$$
\underline{u}(x) \leq u(x) \leq \bar{u}(x) .
$$

Furthermore, the continuity of $f$ and (5) ensure that $u+h(u)$ is continuous. Thus, $u$ is continuous. 
We proceed now with the claim. To see that $\bar{u}$ is a supersolution, take into account that

$$
-\int_{\Omega} J(x-y) \bar{u}_{n}(y) d y+\bar{u}_{n}(x)+h\left(\bar{u}_{n}(x)\right)=\lambda+h(\lambda w(x)) \geq \lambda+h(0)
$$

while on the other hand

$$
f(x)+\int_{\mathbb{R}^{N} \backslash \Omega} J(x-y) g(y) d y \leq\|f\|_{L^{\infty}(\Omega)}+\|J\|_{L^{\infty}\left(\mathbb{R}^{N}\right)}\|g\|_{L^{1}\left(\mathbb{R}^{N} \backslash \Omega\right)} .
$$

Taking $\lambda$ large this shows that $\bar{u}$ is a supersolution. The fact that $\underline{u}$ is a subsolution follows in the same way.

We are almost ready to characterize existence of large solutions. Let us state first a regularity result, which follows with similar arguments that prove Theorem 1.1.

Lemma 3.3. If $u$ is a large solution of $(1)$, then $u \in L^{1}(\Omega)$.

Proof of Theorem 1.2. In order to prove the existence of a large solution, let us perform the following truncation on the function $f$

$$
f_{n}(x)= \begin{cases}f(x) & \text { if } f(x) \leq n, \\ n & \text { if } f(x)>n\end{cases}
$$

and define $u_{n}$ as a solution to

$-\int_{\Omega} J(x-y) u_{n}(y) d y+u_{n}(x)+h\left(u_{n}(x)\right)=f_{n}(x)+\int_{\mathbb{R}^{N} \backslash \Omega} J(x-y) g(y) d y$.

Note that $\left\{u_{n}\right\}$ is a family of continuous and bounded functions. Furthermore, by the comparison stated in Lemma $3.1,\left\{u_{n}\right\}$ is an increasing family in $n$.

In fact, we will see that $u_{n}$ is uniformly bounded from above. With this purpose in mind we construct a family of supersolutions to (12), which are bounded uniformly in $n$.

Declare

$$
\bar{u}_{n}(x):=H\left(f_{n}(x)\right)+\lambda w,
$$

where $\lambda$ is a positive parameter, $w$ is the solution to (11) and $H$ is defined in (7). Let us show that $\bar{u}_{n}$ is the desired supersolution, namely

$$
-\int_{\Omega} J(x-y) \bar{u}_{n}(y) d y+\bar{u}_{n}(x)+h\left(\bar{u}_{n}(x)\right) \geq f_{n}(x)+\int_{\mathbb{R}^{N} \backslash \Omega} J(x-y) g(y) d y
$$

by choosing $\lambda$ sufficiently large. Indeed, assumption (8) yields

$$
\int_{\Omega} J(x-y) H\left(f_{n}(y)\right) d y \leq\|J\|_{L^{\infty}(\Omega)} \int_{\Omega}\left|H\left(f_{n}(y)\right)\right| d y \leq K .
$$


Recalling that $g \in L^{1}\left(\mathbb{R}^{N} \backslash \Omega\right)$, we have that (13) is fulfilled if

$$
-K+\lambda+H\left(f_{n}(x)\right)+h\left(H\left(f_{n}(x)\right)+\lambda w(x)\right) \geq f_{n}(x)+C_{g} .
$$

Whenever $x$ is taken far away from the boundary, this inequality is trivial, for $\lambda$ large, independent of $n$, (notice that $f_{n}(x)=f(x)$ for $n$ large and $x$ away from $\partial \Omega$ ). As $x$ approaches the boundary, notice that

$$
H\left(f_{n}(x)\right)+h\left(H\left(f_{n}(x)\right)+\lambda w(x)\right)-f_{n}(x) \geq 0,
$$

thus the above inequality holds taking $\lambda>C_{g}+K$.

Furthermore, applying the comparison principle we infer that

$$
u_{n}(x) \leq \bar{u}_{n}(x) \leq \bar{u}:=H(f(x))+\lambda w .
$$

As a result, the increasing family $\left\{u_{n}\right\}$ is uniformly bounded by $\bar{u}$, and we can define the pointwise limit

$$
u(x)=\lim _{n \rightarrow \infty} u_{n}(x) .
$$

Monotone convergence returns easily that $u$ is a solution of (1), which

$$
u \leq H(f(x))+\lambda w .
$$

To see that indeed, $u$ is a large solution, we compare it with an appropriate subsolution. Define

$$
\underline{u}_{n}(x):=H\left(\delta f_{n}(x)\right)-\lambda w(x),
$$

where $w$ is once more the nonnegative solution to Problem (11) and $\delta>0$ will be conveniently chosen.

In order to show that $\underline{u}_{n}$ is a subsolution, we need to verify

$$
-\int_{\Omega} J(x-y) \underline{u}_{n}(y) d y+\underline{u}_{n}(x)+h\left(\underline{u}_{n}(x)\right)-f_{n}(x)-\int_{\mathbb{R}^{N} \backslash \Omega} J(x-y) g(y) d y \leq 0 .
$$

The first two terms can be estimated thanks to the uniform boundedness of $f_{n}$ from below. In consequence, there exists a constant $K$ independent on $n$ such that

$$
-\int_{\Omega} J(x-y) \underline{u}_{n}(y) d y+\underline{u}_{n}(x) \leq K-\lambda+H\left(\delta f_{n}(x)\right) .
$$

With respect to the third term, recall that $h$ is increasing, hence $h\left(\underline{u}_{n}(x)\right) \leq$ $h\left(H\left(\delta f_{n}(x)\right)\right)$. Thus, taking into account that $g \in L^{1}\left(\mathbb{R}^{N} \backslash \Omega\right)$ and the former considerations, the inequality (15) is equivalent to

$$
C_{g}+K-\lambda+H\left(\delta f_{n}(x)\right)+h\left(H\left(\delta f_{n}(x)\right)\right)-f_{n}(x) \leq 0 .
$$

As before, by choosing the parameter $\lambda$ large enough, the above inequality holds away from the boundary. Close to the boundary we claim that it is possible to find an appropriate value for $\delta>0$, independent of $n$, such that

$$
H\left(\delta f_{n}(x)\right)+h\left(H\left(\delta f_{n}(x)\right)\right)-f_{n}(x) \leq 0 .
$$


Taking $\lambda>C_{g}+K$ the function $\underline{u}_{n}$ is a subsolution and accordingly by comparison, $u_{n} \geq \underline{u}_{n}$. Recalling that $\left\{u_{n}\right\}$ is an increasing sequence, we indeed obtain that

$$
u(x) \geq u_{n}(x) \geq \underline{u}_{n}(x) .
$$

Now we prove the claim arguing by contradiction. Suppose that

$$
\limsup _{s \rightarrow \infty}(H(\delta s)+h(H(\delta s))-s)=2 L>0,
$$

which implies that there exists a sequence, $s_{j} \rightarrow \infty$, such that

$$
H\left(\delta s_{j}\right)+h\left(H\left(\delta s_{j}\right)\right)-s_{j}>L .
$$

If $H(s)=s$, the above inequality reads as

$$
h\left(\delta s_{j}\right)-(1-\delta) s_{j} \geq L,
$$

or equivalently

$$
\frac{h\left(\delta s_{j}\right)}{\delta s_{j}}-\frac{1-\delta}{\delta} \geq \frac{L}{\delta s_{j}}
$$

Since $h(s) / s<C$ we get the desired contradiction taking $\delta=1 /(1+C)$. Then, by comparison

$$
u_{n}(x) \geq \frac{1}{1+C} f_{n}(x)-\lambda w(x) .
$$

Passing to the limit in (16) shows that

$$
u(x) \geq \frac{1}{1+C} f(x)-\lambda w(x) .
$$

If on the contrary $H(s)=h^{-1}(s)$, inequality (17) can be expressed as

$$
h^{-1}\left(\delta s_{j}\right)-(1-\delta) s_{j} \geq L .
$$

Recall that $h^{-1}(t) / t \rightarrow 0$ as $t$ goes to infinity, which contradicts the previous inequality taking $\delta \in(0,1)$. The comparison result implies that

$$
u_{n}(x) \geq h^{-1}\left(\delta f_{n}(x)\right)-\lambda w(x) \quad \text { for } \delta \in(0,1) .
$$

Making $\delta \rightarrow 1$, we obtain the inequality

$$
u(x) \geq u_{n}(x) \geq h^{-1}\left(f_{n}(x)\right)-\lambda w(x),
$$

which as $n \rightarrow \infty$ reads

$$
u(x) \geq h^{-1}(f(x))-\lambda w(x) .
$$

We conclude the proof looking for a nonexistence result. Arguing by contradiction, if we assume that there exists a large solution the comparison Lemma (see Remark 3.1) implies that the lower estimate (16) holds. Thus, if (8) does not occur then $u \notin L^{1}(\Omega)$ and no large solution exists, see Lemma 3.3 .

Remark 3.2. Let us observe that if $v$ is a large solution, then $u_{n} \leq v$ and passing to the limit $u \leq v$. Thus, $u$ is the minimal large solution. 
Proof of Theorem 1.3. For the minimal large solution, the estimate (9) is deduced directly from (14), (18) and (19).

For a general large solution $v \in L^{1}\left(\mathbb{R}^{n}\right)$ the lower estimate follows by the definition of minimal large solution

$$
v \geq u \geq H(\delta f)-C_{1} .
$$

In order to prove the upper estimate we define

$$
\Omega_{\varepsilon}=\{x \in \Omega: \operatorname{dist}(x, \partial \Omega)>\varepsilon\},
$$

$f_{\varepsilon} \in C\left(\Omega_{\varepsilon}\right)$ such that $f_{\varepsilon}(x) \geq f(x)$ in $\Omega_{\varepsilon}, f_{\varepsilon} \rightarrow f$ as $\varepsilon \rightarrow 0$ and

$$
\lim _{x \rightarrow \partial \Omega_{\varepsilon}, x \in \Omega_{\varepsilon}} f_{\varepsilon}(x)=+\infty, \quad \quad \int_{\Omega_{\varepsilon}} H\left(f_{\varepsilon}(x)\right) d x \leq K<\infty .
$$

We consider the problem

$$
-\int_{\Omega_{\varepsilon}} J(x-y) u_{\varepsilon}(y) d y+u_{\varepsilon}(x)+h\left(u_{\varepsilon}(x)\right)=f_{\varepsilon}(x)+\int_{\mathbb{R}^{n} \backslash \Omega_{\varepsilon}} J(x-y) v(y) d y .
$$

Since $v \in L^{1}\left(\mathbb{R}^{n}\right)$ and

$$
\int_{\mathbb{R}^{n} \backslash \Omega_{n}} J(x-y) v(y) d y \leq\|J\|_{L^{\infty}\left(\mathbb{R}^{n}\right)}\left(\|v\|_{L^{1}(\Omega)}+\|g\|_{L^{1}\left(\mathbb{R}^{n} \backslash \Omega\right)}\right):=C_{v},
$$

according to Theorem 1.2, if we take $\lambda>K+C_{v}$ there exists a minimal solution of (20) satisfying

$$
\begin{cases}u_{\varepsilon}(x) \leq H\left(f_{\varepsilon}(x)\right)+\lambda w(x) & x \in \Omega_{\varepsilon}, \\ u(x)=v(x) & x \in \mathbb{R}^{n} \backslash \Omega_{\varepsilon} .\end{cases}
$$

Furthermore, since $f_{\varepsilon}(x) \geq f(x)$ in $\Omega_{\varepsilon}$ it is easy to see that $v$ is indeed a subsolution to $(20)$ in $\Omega_{\varepsilon}$. Moreover, $v$ is bounded in $\Omega_{\varepsilon}$. Hence, the comparison principle (see Remark 3.1) implies that $v(x) \leq u_{\varepsilon}(x)$ and then

$$
v(x) \leq H\left(f_{\varepsilon}(x)\right)+\lambda w(x) \quad \text { in } \Omega_{\varepsilon} .
$$

Since $H\left(f_{\varepsilon}\right) \rightarrow H(f)$ as $\varepsilon \rightarrow 0$, passing to the limit in the above inequality shows that $v$ verifies $(9)$.

Remark 3.3. Taking

$$
f_{\varepsilon_{1}}(x) \geq f_{\varepsilon_{2}}(x)+\|J\|_{L^{\infty}\left(\mathbb{R}^{n}\right)} K\left|\Omega_{\varepsilon_{2}} \backslash \Omega \varepsilon_{1}\right|, \quad \text { for } \varepsilon_{1}>\varepsilon_{2},
$$

it is easy to prove that $u_{\varepsilon}$ is decreasing in $\varepsilon$. Thus, the maximal large solution can be obtained as the limit of $u_{\varepsilon}$.

\section{UNIQUENESS}

We devote this part of the work to the analysis of uniqueness of large solutions. We start by treating the case $f \geq 0$ and $h(s) / s$ being unbounded and non-decreasing. 
Proof of Theorem 1.4 (1). Suppose that $u, v$ are two solutions to Problem (1). Denote

$$
\mathcal{A}:=\{x \in \Omega \text { such that } u(x)>v(x)\},
$$

and assume that $\mathcal{A} \neq \emptyset$. Thus, there exists $k>1$ for which the set

$$
\mathcal{A}_{k}:=\left\{x \in \Omega \text { such that } w_{k}(x):=u(x)-k v(x)>0\right\} \neq \emptyset .
$$

The blow-up rates in Theorem 1.3 imply that

$$
\lim _{x \rightarrow \partial \Omega, x \in \Omega} \frac{w_{k}(x)}{H(f(x))}=1-k<0 .
$$

Hypothesis (6) guarantees that $H(f)>0$ close to boundary (indeed, $H(f)=$ $+\infty$ on $\partial \Omega$ ). Accordingly, $w_{k}<0$ approaching the boundary, thus $\mathcal{A}_{k} \subset \subset \Omega$. Furthermore, there exists $\hat{x} \in \Omega$ such that $w_{k}(\hat{x})=\max _{\overline{\mathcal{A}_{k}}} w_{k}$. The fact that $w_{k} \leq 0$ in $\mathcal{A}_{k}^{c}$ entails that $\hat{x} \in \mathcal{A}_{k}$ and then $w_{k}(\hat{x})=\max _{\mathbb{R}^{N}} w_{k}$.

On the other hand, recall that $h(s)$ is increasing and we are considering that $h(s) / s$ is nondecreasing. In this case it holds that

$$
k h(v(x)) \leq h(k v(x)) \leq h(u(x)) \quad x \in \mathcal{A}_{k} .
$$

Evaluate now at $\hat{x}$ the equation for $w_{k}$ and use the positivity of $f$ to get

$-\int_{\mathbb{R}^{N}} J(\hat{x}-y)\left(w_{k}(y)-w_{k}(\hat{x})\right) d y=-h(u(\hat{x}))+k h(v(\hat{x}))+(1-k) f(\hat{x}) \leq 0$

However, $\hat{x}$ is a point of maximum hence

$$
-\int_{\mathbb{R}^{N}} J(\hat{x}-y)\left(w_{k}(y)-w_{k}(\hat{x})\right) d y \geq 0 .
$$

These inequalities imply that $w_{k}$ is a constant function, which is a contradiction with the fact that $w_{k}(\hat{x})>0$ and $w_{k}<0$ close to the boundary.

If we assume that the kernel $J$ is symmetric, fact that allows an integration by parts in our equation, we prove uniqueness without extra hypothesis on $h$ and $f$.

Proof of Theorem 1.4 (2). Recall that by Lemma 3.3 any large solution must belong to $L^{1}(\Omega)$. Uniqueness is then a direct consequence of the following version of the comparison principle for sub and supersoluions in $L^{1}(\Omega)$.

Lemma 4.1. Assume that $J$ is symmetric. Let $\bar{u}$ and $\underline{u}$ be a classical $L^{1}(\Omega)$ supersolution and subsolution of (1), respectively. Then, $\underline{u} \leq \bar{u}$.

Proof. The function $w(x)=\underline{u}(x)-\bar{u}(x)$ satisfies

$$
-\int_{\Omega} J(x-y)(w(y)-w(x)) d y+w(x) \int_{\mathbb{R}^{N} \backslash \Omega} J(x-y) d y+h(\underline{u}(x))-h(\bar{u}(x)) \leq 0,
$$


Multiplying by $z_{M}(x)=\min \left\{w^{+}(x), M\right\}$ where $w_{+}=\max \{0, w\}$ and integrating in $\Omega$ gives

$$
I_{1}+I_{2}+I_{3} \leq 0
$$

being

$$
\begin{aligned}
& I_{1}=-\int_{\Omega} z_{M}(x) \int_{\Omega} J(x-y)(w(y)-w(x)) d y d x \\
& I_{2}=\int_{\Omega} z_{M}(x) w(x) \int_{\mathbb{R}^{N} \backslash \Omega} J(x-y) d y d x, \\
& I_{3}=\int_{\Omega}(h(\underline{u}(x))-h(\bar{u}(x))) z_{M}(x) d x .
\end{aligned}
$$

Using the hypothesis that $J$ is symmetric and since $z_{M} \in L^{\infty}(\Omega)$, we get that

$$
I_{1}=\frac{1}{2} \int_{\Omega} \int_{\Omega} J(x-y)(w(y)-w(x))\left(z_{M}(y)-z_{M}(x)\right) d y d x .
$$

Recall that the integrands in $I_{2}$ and $I_{3}$ are nonnegative, as well as the function $T(x, y)=(w(y)-w(x))\left(z_{M}(y)-z_{M}(x)\right)$. As a result the three integrals above must vanish. From $I_{3}=0$ we infer that $z_{M} \equiv 0$, hence $w^{+} \equiv 0$. This implies that $w \leq 0$ as desired.

\section{Breakage of the Keller-Osserman condition for integrable SYMMETRIC KERNELS}

We conclude this work showing that, even when we allow the kernel to be singular at the origin, it is still not possible to reach a balance between this nonlocal diffusion and any absorption term, to obtain large solutions, without the action of an explosive source.

More precisely we consider Problem (1) with a probability density which is symmetric, singular at the origin and it satisfies $J(x)>\alpha>0$ in a small ball centered at the origin.

As we already observed in Section 2, if a large solution exists, it must be in $L^{1}(\Omega)$. However, since $J$ is just integrable, the term $J * u$ in equation (1) is not necessarily continuous, hence neither the solution is automatically continuous.

On the other hand, since $J * u \in L^{1}(\Omega)$ if we admit that $f \in L^{\infty}(\Omega)$ yields that $h(u) \in L^{1}(\Omega)$. Arguing now as in Lemma 4.1 we obtain a comparison principle and consequently, uniqueness of $L^{1}(\Omega)$ solutions.

At this stage, it is not difficult to see that if $h(s) \rightarrow \pm \infty$ as $s \rightarrow \pm \infty$, then $\underline{u}=-K$ and $\bar{u}=K$ are a sub and a supersolution to Problem (1), respectively. The sub-super solutions method guarantees the existence of a bounded weak solution. By uniqueness, no large solution exists.

Acknowledgements: R. Ferreira is supported by project MTM2014-53037P (Spain). M. Pérez-Llanos is supported by proyects ANPCYT PICT20141771 and CONICET PIP $11220150100032 \mathrm{CO}$ (Argentine). This research 
was accomplished while M. Pérez-Llanos was visiting U. Complutense de Madrid. She feels really grateful to many people of this institution for their hospitality and nice atmosphere. The authors would like to express their gratitud to the referees for some interesting suggestions that improved and enhanced the former version of this manuscript.

\section{REFERENCES}

[1] C. Bandle,and M. Marcus, Large solutions of semilinear elliptic equations: existence, uniqueness and asymptotic behaviour. Festschrift on the occasion of the 70th birthday of Shmuel Agmon. J. Anal. Math. 58 (1992), 9-24.

[2] H. Chen, P. Felmer, and A. Quaas, Large solutions to elliptic equations involving fractional Laplacian. Ann. Inst. H. Poincar Anal. Non Linéaire 326 (2015), 11991228 .

[3] J. García-Melián and J. C. Sabina de Lis, A boundary blow-up problem with a nonlocal reaction, Nonlinear Analysis 75, (2012), 2774-2792.

[4] J. García-Melián and J. D. Rossi, A logistic equation with refuge and nonlocal diffusion. Commun. Pure Appl. Anal. 86 (2009), 2037-2053.

[5] J. García-Melián and J. D. Rossi, On the principal eigenvalue of some nonlocal diffusion problems. J. Differential Equations 246 (2009), no. 1, 21-38

[6] J. B. Keller, On solutions of $\Delta u=f(u)$. Comm. Pure Appl. Math. 10, (1957), 503-510

[7] J. López-Gómez, Metasolutions of Parabolic Equations in Population Dynamics, Taylor \& Francis Group, 2016.

[8] J. López-Gómez, On the structure and stability of the set of solutions of a nonlocal problem modeling Ohmic heating. J. Dynam. Differential Equations 10, (1998), no. 4, $537-566$

[9] R. Osserman, On the inequality $\Delta u \geq f(u)$. Pacific J. Math. 7, (1957), 1641-1647

[10] J. D. Rossi and E. Topp, Large solutions for a class of semilinear integro-differential equations with censored jumps. J. Differential Equations 260, (2016), no. 9, 68726899.

[11] L. Véron, Singularities of solutions of second order quasilinear equations. Pitman Research Notes in Mathematics Series, 353. Longman, Harlow, 1996.

RAÚL FERREIRA

Departamento de Matemática Aplicada,

Fac. De C.C. Químicas, U. Complutense de Madrid, 28040, MADRID, SPAIN.

E-mail address: raul_ferreira@mat.ucm.es

MAYte PÉREZ-Llanos

Instituto de Investigaciones Matemáticas Luis Santaló (IMAS) And CONICET

Facultad de Ciencias Exactas y Naturales, U. De Buenos Aires

Ciudad Universitaria Pabellón I,

C1428EgA - Buenos Aires, Argentine.

maytep@dm.uab.ar 